Jochen Brack

\title{
Der Einsatz von Buprenorphin in der stationären Entzugsbehandlung
}

Eine Anwendungsbeobachtung

Use of Buprenorphine within In-Patient Detoxification - a Monitoring of its Application

\section{Zusammenfassung}

Im Rahmen der stationären Entgiftung einer schwerpunktmäßig auf Therapievorbereitung orientierten Drogenentzugsstation wurden bei 60 Patienten die Entzugsbehandlung mit Buprenorphin gegen die mit Methadon bzw. Levomethadon verglichen. 30 Patienten waren monoabhängige Heroinkonsumenten, $30 \mathrm{~Pa}$ tienten entzogen von zum Teil langjähriger Methadonsubstitution, so dass vier Vergleichgruppen à 15 Patienten gebildet wurden. Es zeigte sich eine, gemessen an Dauer und Ausprägung der Entzugssymptome deutliche Überlegenheit von Buprenorphin in der Entzugsbehandlung von langjährigen Substitutionspatienten. Depressive Verstimmungszustände im Gesamtverlauf der Entgiftung treten unter Buprenorphin seltener auf als unter der Gabe von Methadon. Bei der Entgiftung von reinem Heroinkonsum scheint Buprenorphin nur bezüglich der Klarheit bzw. des raschen Wegfalls psychischer Abschirmung überlegen, die typische Entzugssymptomatik wurde bei einigen Symptomen besser von Buprenorphin bei anderen besser von Methadon beeinflusst, wobei die Unterschiede gering blieben.

\section{Schliuisselwörter}

Buprenorphin · stationäre Entzugsbehandlung · Methadonsubstitution · Opiatabhängigkeit

\section{Abstract}

Buprenorphine-assisted detoxification was compared to methadone- respectively l-methadone-assisted detoxification within a detoxification program emphasizing treatment preparation. In total 60 patients were included, either half was detoxified from heroin $(n=30)$ or methadone maintenance $(n=30)$ respectively. Finally, four study groups were established (heroin/buprenorphine, heroin/methadone, methadone/buprenorphine, methadone/methadone). Considering withdrawal symptoms, buprenorphine was superior to methadone in long-term methadone maintenance patients. Depression was found to be less frequent in patients detoxified by buprenorphine compared to methadone. Regarding the heroin group, buprenorphine was superior only with regard to cognitive distinctness. As to control of withdrawal symptoms, differences between buprenorphine and methadone were only marginal.

Key words

Buprenorphine · in-patient detoxification · methadone maintenance treatment $\cdot$ opiate addiction 
Im Gegensatz zum Methadon (bzw. Polamidon), bei dem es sich um einen reinen $\mu$-Rezeptor-Agonisten handelt, verfügt das $\mathrm{Bu}-$ prenorphin über eine gemischte Rezeptoraffinität. Das Buprenorphin ist ein partieller Agonist am $\mu$-Opioidrezeptor und führt somit zu Euphorie, Analgesie und Atemdepression.

Auf der anderen Seite handelt es sich bei Buprenorphin um einen Antagonisten am $\kappa$-Opioidrezeptor, der verantwortlich ist für Sedierung und Dysphorie. Im Umkehrschluss bedeutet dies, dass das Buprenorphin am $\kappa$-Rezeptor als Antagonist für antidepressive Effekte verantwortlich gemacht wird [1 -4]. Es wird von aufklarenden Eigenschaften und der Wiederkehr von Emotionen berichtet [5]. Des Weiteren werden dem Buprenorphin auch antipsychotische Effekte nachgesagt [6].

Methadon bringt hingegen vor allem am $\mu$-Rezeptor die euphorische, analgetische und atemdepressive Wirkung hervor und ist als $\kappa$-Rezeptoragonist vermutlich für Dysphorie und Sedierung verantwortlich zu machen.

Als partieller Agonist erreicht das Buprenorphin auch bei sehr hoher Dosierung nur submaximale Wirkung und dann ein Plateau, über das hinaus eine Dosissteigerung wirkungslos ist (Ceiling-Effekt). Aus diesem Grunde weist Buprenorphin eine höhere therapeutische Breite auf, so dass Überdosierungen nur sehr schwer möglich sind [3, 7-11]. An Rhesusaffen konnte nachgewiesen werden, dass Buprenorphin auch bei hohen Dosierungen (10 mg/kg) keine Atemdepression verursacht [12].

Die hohe Affinität des Buprenorphin zum $\mu$-Rezeptor gilt als weitgehend irreversibel, agonistische Effekte können also durch Antagonisten nur schwer aufgehoben werden. Buprenorphin verdrängt andere Opiate aus der Rezeptorbindung und blockiert zusätzlich eingenommene Opiate in ihrer Wirkung [13]. Aus diesem Grunde kann es bei der Verabreichung von Buprenorphin bei einem Patienten, der Heroin oder L-Polamidon bzw. Methadon eingenommen hat, zu entsprechenden Entzugsymptomen kommen. Deshalb wird empfohlen Buprenorphin erst zu verabreichen, wenn die genannten Substanzen weitgehend aus dem Körper eliminiert sind, bzw. die ersten Entzugssymptome auftreten [14-17].

$\mathrm{Zu}$ den weiteren pharmakologischen Daten von Buprenorphin siehe zusammenfassend Tab $\mathbf{1}$.

\section{Anwendungsbeobachtung}

Umfangreiche Vergleichsuntersuchungen über die Entzugsbehandlung von Opiatabhängigen mit Buprenorphin versus Methadon bzw. Levomethadon liegen nicht vor, da solche Studien von den Herstellern nicht finanziert werden und wir entsprechend auch kein Studiendesign und über keine übliche Forschungsforderungen erfüllende Auswertung neben unserer anstrengenden praktischen Versorgungstätigkeit erarbeiten konnten.

In einer Studie von Bickel et al. wurde Buprenorphin bei 45 heroinabhängigen Patienten in der Detoxifikation mit Methadon verglichen. Als Ergebnis zeigte sich, dass Buprenorphin im Ver-
Tab. 1 Pharmakologische Daten von Buprenorphin

\begin{tabular}{|c|c|}
\hline $\begin{array}{l}\text { Sublingualtabletten mit den } \\
\text { Dosierungen: } \\
\text { Wirkdauer (klinisch): }\end{array}$ & $\begin{array}{l}0,4 \mathrm{mg}, 2 \mathrm{mg}+8 \mathrm{mg} \\
24 \text { bis } 72 \mathrm{std} .\end{array}$ \\
\hline $\begin{array}{l}\text { Plasmaproteinbindung: } \\
\text { relative Bioverfügbarkeit: }\end{array}$ & $\begin{array}{l}\text { ca. } 96 \% \alpha \text { - und } \beta \text {-Globuline, nicht Albumin } \\
\text { sl Tabletten } 50 \text { bis } 70 \%\end{array}$ \\
\hline \multicolumn{2}{|c|}{$\begin{array}{l}\text { biliär und renal, } 70 \% \text { fäkale Ausscheidung, hohe Lipophilie, langsame Freiset- } \\
\text { zung aus dem Fettgewebe }\end{array}$} \\
\hline \multicolumn{2}{|c|}{ Als Nebenwirkungen sind beschrieben: } \\
\hline \multicolumn{2}{|c|}{$\begin{array}{l}\text { Schwindel, Kopfschmerzen, Miosis, Übelkeit, Verstopfung, Schweißausbruch } \\
\text { oder Erbrechen. } \\
\text { Bei gleichzeitiger Gabe von Ketokonazol (Antimikotikum), Cimetidin (H2 } \\
\text { Blocker) und Erythromycin (Antibiotikum) kann der Abbau von Buprenorphin } \\
\text { gehemmt werden, d. h. es kommt zu einer Wirkungsverstärkung. } \\
\text { Carbamazepine führen zu einem beschleunigten Abbau von Buprenorphin, } \\
\text { d. h. es kommt zu einer Wirkungsabschwächung. } \\
\text { Eine HIV-Therapie mit Proteaseinhibitoren (Ritonavir, Indinavir oder Saquinavir) } \\
\text { führt zu einer Wirkabschwächung von Buprenorphin (in vivo). Umgekehrt be- } \\
\text { steht keine Einflussnahme der Proteaseinhibitorenspiegel durch Buprenorphin } \\
{[18,19]}\end{array}$} \\
\hline
\end{tabular}

gleich zu Methadon von den Patienten gut vertragen wurde. Bei Buprenorphin zeigte sich ein Trend zu einer besseren Haltequote [20-23].

Mit den von Paetzold et al., Scheider et al., O-Condor, Nigam et al., Cheskin et al. und Janiri et al. [24-28] vorgelegten Vergleichsuntersuchungen zu Buprenorphin versus Clonidin bzw. Oxazepam und Carbamazepin, ist in unserer Praxis nichts anzufangen, da nach unseren Erfahrungen in der klinischen Praxis nachgewiesen ist, dass die homologe Entzugsbehandlung der Opiate eindeutig überlegen ist und insbesondere die Haltequote bei einer Entzugsbehandlung mit Clonidin oder Oxazepam gering ist.

Wir wollten mit den uns zur Verfügung stehenden Mitteln unsere klinische Erfahrung auf eine bessere Grundlage stellen und bitten die Ergebnisse auch nur in diesem Sinne zu verstehen. Wir begannen Mitte des ten auf unserenJahres 2000 Buprenorphin (Subutex ${ }^{\circledR}$ ) in der Entzugsbehandlung von opiatabhängigen Drogenpatien vier Drogenentzugsstationen (à 15 Betten) einzusetzen. Auf der Station mit dem Schwerpunkt der Vorbereitung auf eine sich anschließende stationäre Entwöhnungsbehandlung, führten wir einen Vergleich zwischen Methadon- bzw. Polamidon ${ }^{\circledR}$-Entzügen und Subutex ${ }^{\circledR}$-Entzügen durch. Insgesamt wurden 60 Patienten in diese Untersuchung einbezogen, wobei die folgenden zwei Gruppen gebildet wurden:

- Heroinkonsumenten, die nur vom Heroin zu entziehen waren (Monoabhängige).

- Langjährig Methadon- bzw. Polamidonsubstituierte, die zur Entgiftung von Methadon bzw. Polamidon in unsere stationäre Behandlung kamen und keinen Beikonsum aufwiesen, der eine zusätzliche Medikation erforderlich machte.

In diesen zwei Gruppen wurde jeweils die Hälfte der Patienten mit Methadon oder L-Polamidon ${ }^{\circledR}$ entzogen, die andere Hälfte wurde mit Subutex ${ }^{\circledR}$ entgiftet. 


\section{Entzugsschemata}

Bei einer Entzugsbehandlung von Heroin mit Methadon bzw. Polamidon wurden am ersten Tag $2 \times 1 \mathrm{ml}$ Methadon (=10 mg) oder Polamidon $^{\circledR}$ (= $5 \mathrm{mg}$ ) verabreicht und ab dem zweiten Tag des stationären Aufenthaltes alternierend eine tägliche Reduktion um vier Tropfen (= $1 \mathrm{mg}$ ) durchgeführt.

Bei der Entzugsbehandlung von Methadon oder Polamidon ${ }^{\circledR}$ mit Methadon oder Polamidon ${ }^{\circledR}$ erfolgte am zweiten Tag eine Verminderung der Ausgangssubstitutionsmenge um $1 \mathrm{ml}$ und dann eine weitere tägliche Reduktion um jeweils $0,5 \mathrm{ml}$ bis auf $2 \mathrm{ml}$. Danach wurde entsprechend wie oben dargestellt um täglich vier Tropfen $(=1 \mathrm{mg})$ bis auf Null reduziert.

Bei der Entzugsbehandlung bei reinen Heroinkonsumenten mit Subutex ${ }^{\circledR}$ wurde nach einem Schema vorgegangen, wie es in der Tab. 2 dargestellt ist.

Tab. 2 Entzugsschemata Subutex ${ }^{\circledR}$

\begin{tabular}{|lll}
\hline bei Heroinkonsum & $\begin{array}{l}\text { bei Methadon-/ } \\
\text { Polamidonkonsum }\end{array}$ \\
\hline 1. Tag & $2 \times 4 \mathrm{mg}$ & $2 \times 8 \mathrm{mg}$ \\
\hline 2. Tag & $2 \times 4 \mathrm{mg}$ & $2 \times 8 \mathrm{mg}$ \\
\hline 3. Tag & $4 \mathrm{mg}-0-2 \mathrm{mg}$ & $8 \mathrm{mg}-0-6 \mathrm{mg}$ \\
\hline 4. Tag & $2 \times 2 \mathrm{mg}$ & $6 \mathrm{mg}-0-7,2 \mathrm{mg}$ \\
\hline 5. Tag & $2 \mathrm{mg}-0-1,2 \mathrm{mg}$ & $6 \mathrm{mg}-0-6 \mathrm{mg}$ \\
\hline 6. Tag & $2 \times 1,2 \mathrm{mg}$ & $2 \times 4 \mathrm{mg}$ \\
\hline 7. Tag & $1,2 \mathrm{mg}-0-0,4 \mathrm{mg}$ & $4 \mathrm{mg}-0-2 \mathrm{mg}$ \\
\hline 8. Tag & $0,4 \mathrm{mg}-0-0,4 \mathrm{mg}$ & $2 \times 2 \mathrm{mg}$ \\
\hline 9. Tag & $0,4 \mathrm{mg}-0-0$ & $2 \mathrm{mg}-0-1,2 \mathrm{mg}$ \\
\hline 10. Tag & abgesetzt & $\begin{array}{l}\text { weiter wie Schema bei } \\
\text { Heroinkonsumenten, sodass } \\
\end{array}$ \\
& & $\begin{array}{l}\text { am } 14 \text {. Tag das Subutex } \\
\text { abgesetzt wurde. }\end{array}$ \\
\hline
\end{tabular}

Bei der Entzugsbehandlung von Polamidon ${ }^{\circledR}$-/Methadonkonsumenten mit Subutex ${ }^{\circledR}$ wurden alle Patienten bis auf $4 \mathrm{ml} \mathrm{Me-}$ thadon/Polamidon ${ }^{\circledR}$ herunterdosiert (tägliche Reduktion der Methadondosis um 0,5 ml) und dann auf Subutex ${ }^{\circledR}$ entsprechend dem Schema in Tab. 2. Sehr wichtig war dabei, dass die erste Gabe des Subutex ${ }^{\circledR} 24$ Std. nach Erhalt der letzten Methadon/Polamidon ${ }^{\circledR}$-Dosis erfolgte, um entsprechend heftige Entzugssymptome zu vermeiden.

\section{Untersuchungsinstrumente}

Eingesetzt wurde ein Untersuchungsbogen, der sich eng anlehnt an einen Bogen, wie er in der Psychiatrischen Universitätsklinik Basel verwandt wurde [26].

Die Patienten wurden jeweils am Morgen eines Tages von der Krankenpflege hinsichtlich der Entzugssymptome entsprechend dem Untersuchungsbogen befragt. Die Bewertung gliederte sich folgendermaßen: 0 = nicht vorhanden, 1 = wenig, 2 = mittel und 3 = stark vorhanden. Im Verlauf der Anwendungsbeobachtung wurde deutlich, dass nicht alle im Untersuchungsbogen aufgeführten Kriterien sich als tauglich erwiesen. Insbesondere die Items Puls, Blutdruck, Tremor, Muskelverspannungen, epileptischer Anfall, Wahnideen, Halluzinationen, Miosis und Mydriasis erwiesen sich als nicht signifikant zur Beschreibung eines Entzugssyndroms bei Opiatabhängigen.

Als Zielparameter der Anwendungsbeobachtung wurden die folgenden Kriterien gewählt:

- erfolgreiche Entgiftung (Haltequote)

- Verlauf der Entzugsbehandlung (Ausprägungsgrad des Entzugssyndroms).

Ergebnisse

Es wurden 30 drogenabhängige Patienten, die während der Entzugsbehandlung Buprenorphin erhielten, mit 30 Patienten, die mit Methadon bzw. L-Polamidon ${ }^{\circledR}$ behandelt wurden, verglichen. Die demographischen Daten (Geschlecht, Schulbildung, Berufsausbildung und Alter) zeigten in den verschiedenen Gruppen keine wesentlichen signifikanten Unterschiede (siehe Tab. 3). Bei Bildung einer Gesamtsumme aller Entzugssymptome pro Tag ergibt sich das folgende Bild:

Im Vergleich von Methadon versus Subutex beim reinen Heroinentzug schneidet das Methadon bzw. Polamidon besser ab, als das Subutex was die Kupierung der Entzugssymptome anbetrifft.

$\mathrm{Zu}$ Beginn der Entzugsbehandlung kommt es beim Methadon zu einem geringer ausgeprägten Entzugssyndrom als beim Subutex, welches bis zum fünften Tag anhält. Danach kommt es am sechsten und siebten Tag zu einem leichten Anstieg der Entzugssymptome auch über das Niveau der mit Subutex entgifteten Patienten und fällt dann am achten Tag wieder unter das Niveau der mit Subutex entgifteten Patienten (siehe Abb. 1).

Bei den Patienten, die vor Beginn der Entgiftung mit Methadon substituiert waren und vom Methadon entziehen wollten, stellt sich ein anderes Bild dar:

Hier erweist sich, dass Subutex dem homologen Entzug mit Methadon als deutlich überlegen (siehe Abb. 2).

Bei Betrachtung der Entzugssymptome im Einzelnen war auffällig, dass bei der Entzugsbehandlung der reinen Heroinabhängigen mit Methadon folgende Entzugssymptome geringer ausgeprägt auftreten als bei der Entgiftung mit Subutex:

Gliederschmerzen, Appetitlosigkeit, Schlafstörungen, Ruhelosigkeit und Dysphorie bzw. Reizbarkeit (siehe Abb. 3).

Hingegen treten folgende Entzugssymptome bei der Entgiftung mit Subutex weniger in Erscheinung:

Schwitzen, Tränen oder Nasenfluss, Gänsehaut, Hitze und Kältegefühl, Durchfall, Bauchkrämpfe, Erbrechen und Verlangen nach Drogen (siehe Abb. 4). 
Tab. 3 Demographische Daten der Stichprobe

\begin{tabular}{|c|c|c|c|c|}
\hline Fallgruppe & $\begin{array}{l}\text { Entgiftung von Heroin } \\
\text { mit Methadon } n=15\end{array}$ & $\begin{array}{l}\text { Entgiftung von Heroin } \\
\text { mit Subutex }^{\circledR} n=15\end{array}$ & $\begin{array}{l}\text { Entgiftung von Methadon } \\
\text { mit Methadon } n=15\end{array}$ & $\begin{array}{l}\text { Entgiftung von Methadon } \\
\text { mit Subutex }{ }^{\circledR} n=15\end{array}$ \\
\hline männlich & $60 \%$ & $70 \%$ & $70 \%$ & $60 \%$ \\
\hline weiblich & $40 \%$ & $30 \%$ & $30 \%$ & $40 \%$ \\
\hline Durchschnittsalter & 29 & 34 & 30 & 31 \\
\hline $\begin{array}{l}\text { Heroinkonsumbeginn } \\
\text { (Durchschnitt) }\end{array}$ & 21 & 26 & 18 & 20 \\
\hline Heroinkonsumdauer & 2-10 Jahre & 3-8 Jahre & 5-14 Jahre & 6-15 Jahre \\
\hline Kokainkonsum & $100 \%$ & $93 \%$ & $90 \%$ & $100 \%$ \\
\hline Kokaineinstiegsalter & 20 & 26 & 21 & 22 \\
\hline Methadonsubstitution (Jahre) & - & - & 1-6 Jahre & 1-7 Jahre \\
\hline Methadondosis & - & - & $3-14 \mathrm{ml}$ & $2-17 \mathrm{ml}$ \\
\hline \multicolumn{5}{|l|}{ Ergebnis der Behandlung } \\
\hline Abbruch & $30 \%$ & $26 \%$ & $35 \%$ & $30 \%$ \\
\hline Entgiftung & $70 \%$ & $74 \%$ & $65 \%$ & $70 \%$ \\
\hline
\end{tabular}

Bei Betrachtung der einzelnen Entzugssymptome bei den mit Methadon bzw. Polamidon substituierten Drogenabhängigen stellt sich das folgende Bild dar:

Bei den mit Subutex entgifteten Patienten treten weniger Entzugssymptome im Bereich Hitze- und Kältegefühl, Ruhelosigkeit, Bauchkrämpfe, Schwitzen, Appetitlosigkeit, Dysphorie bzw. Reizbarkeit, Schlafstörungen und Verlangen nach Drogen auf (siehe Abb. 5).

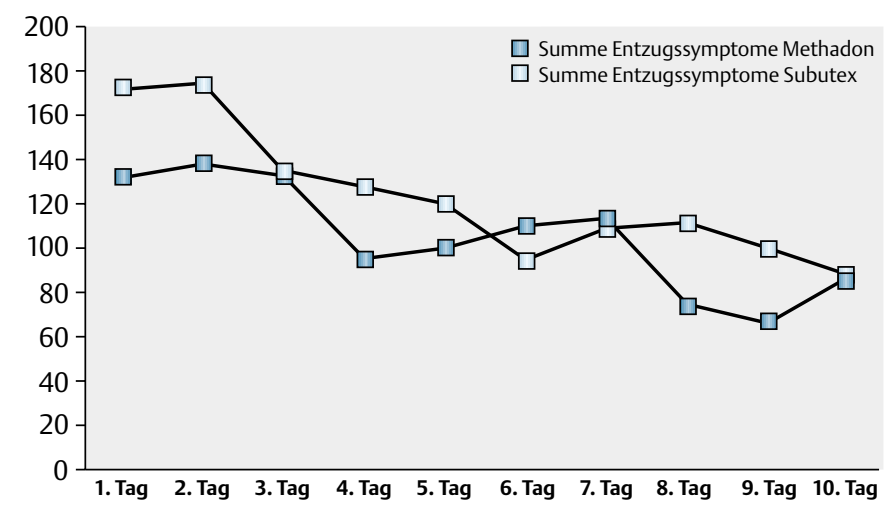

Abb. 1 Vergleich Heroinentzug mit Methadon versus Subutex

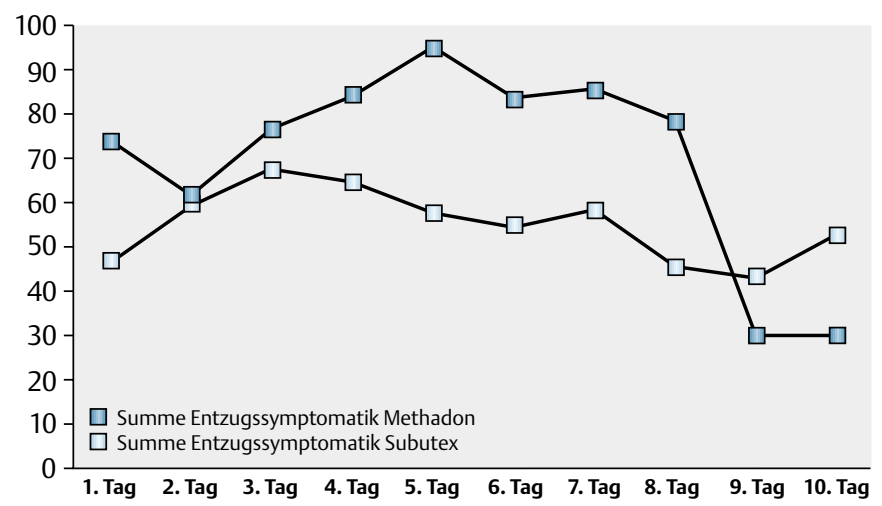

Abb. 2 Vergleich Methadonentzug mit Methadon versus Subutex
Hingegen treten unter der Medikation mit Subutex folgende Entzugssymptome häufiger auf als bei der Gabe von Methadon:

Gliederschmerzen, Durchfall und Erbrechen (siehe Abb. 6).

Gleich häufig treten die folgenden Entzugssymptome auf:

Tränen oder Nasenfluss und Gänsehaut (siehe Abb. 7).

Darüber hinaus gibt es aus den bisherigen klinischen Erfahrungen folgende Beobachtungen bzw. Aussagen der Patienten, die mit Buprenorphin entzogen wurden, zu berichten:

Viele der drogenabhängigen Patienten in den BuprenorphinGruppen berichteten unter Buprenorphin über ein Gefühl der „Klarheit“ bzw. „Wachheit“, welches sie bei früheren Entzügen unter Polamidon ${ }^{\circledR}$ bzw. Methadon so nicht erlebt hatten.

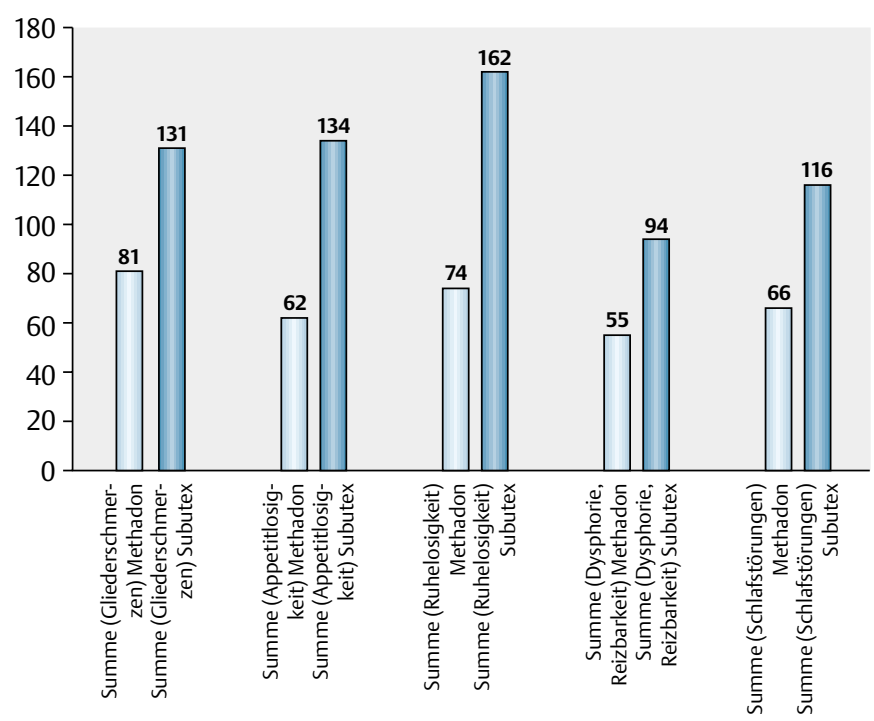

Abb. 3 Entzugssymptome bei Heroinkonsumenten - Subutex versus Methadon 


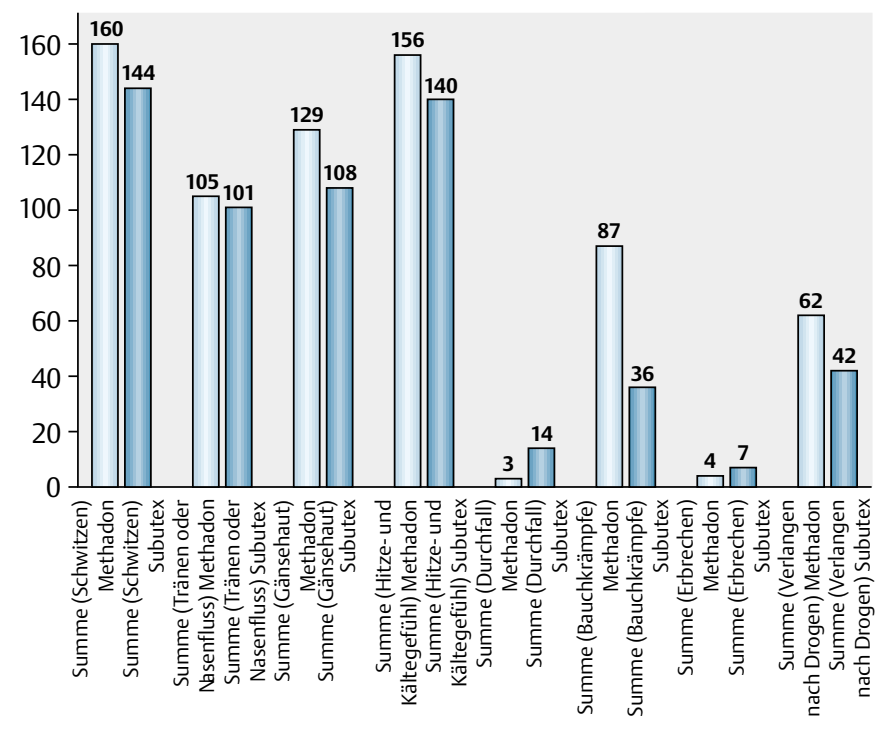

Abb. 4 Entzugssymptome bei Heroinkonsumenten - Subutex versus Methadon

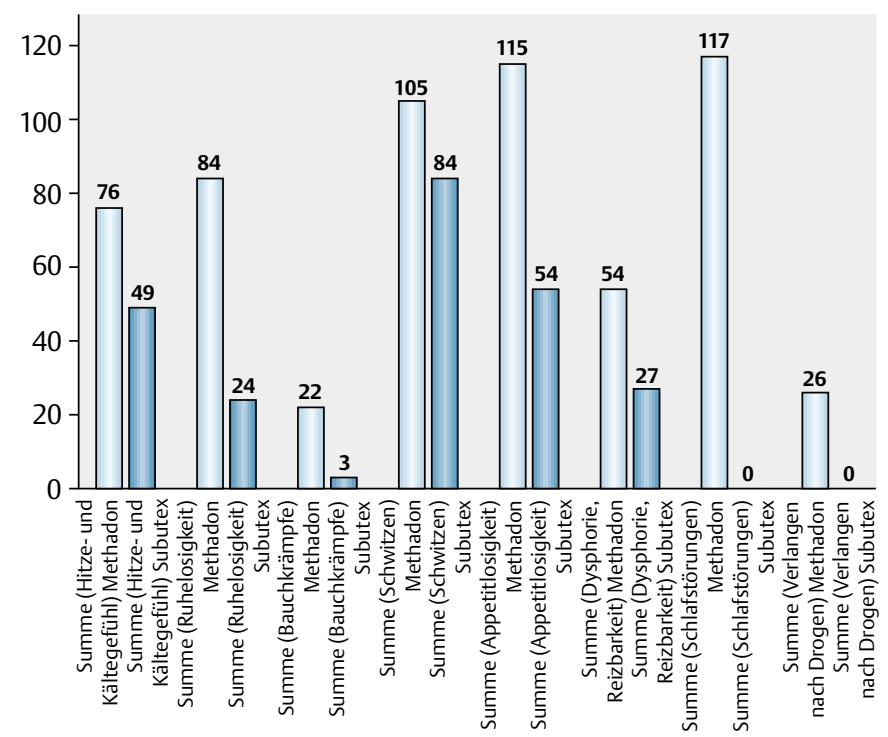

Abb. 5 Entzugssymptome bei Heroinkonsumenten - Subutex versus Methadon

Dies entspricht einer Vergleichsuntersuchung von Buprenorphin und Methadon im Rahmen einer Erhaltungstherapie Opiatkranker [5].

Die Patienten beschrieben darüber hinaus ihren psychischen $\mathrm{Zu}$ stand als deutlich realitätsbezogener als im Rahmen von methadongestützten Entzugsbehandlungen.

Dieses Gefühl von mehr „Klarheit“ und „Wachheit“ wurde von vielen Patienten als sehr angenehm erlebt und führte dazu, dass sie sich besser mit einer anstehenden Langzeitentwöhnungsbehandlung auseinander setzen konnten. Auf der anderen Seite berichteten jedoch auch einige Patienten, dass sie diese Gefühle von Klarheit und Wachheit kaum ertragen können, da sie ihnen als fremd entgegen treten.

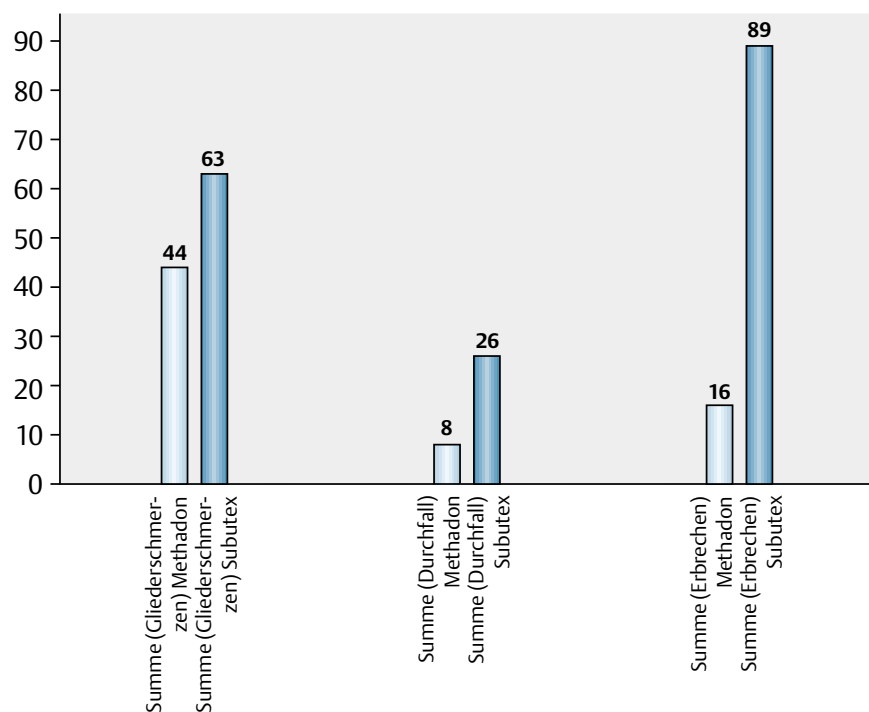

Abb. 6 Entzugssymptome bei Heroinkonsumenten - Subutex versus Methadon

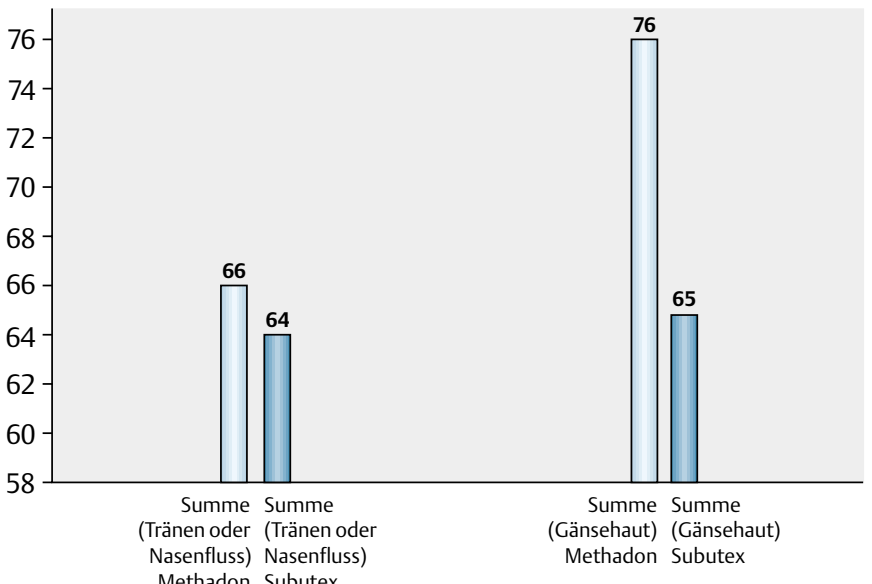

Abb. 7 Entzugssymptome bei Heroinkonsumenten - Subutex versus Methadon

Die klinischen Erfahrungen besagen darüber hinaus, dass zu Beginn der Entgiftungsbehandlung (Induktionsphase) unter Buprenorphin häufiger dysphorische Zustände auftraten, die einhergingen mit einer subjektiv erlebten Unterdosierung. Darauf musste dann häufig mit einer entsprechenden Bedarfsmedikation von Buprenorphin reagiert werden, die bis zu $2 \times 4 \mathrm{mg}$ Subutex zusätzlich täglich betrug. Bis dann die entsprechende Einstiegsdosis bzw. Umstellungsdosis gefunden wurde, konnten ein bis zwei Tage vergehen. Bei entsprechender Dosiserhöhung trat dann schnell ein allgemeines Wohlbefinden auf und mit der Entzugsbehandlung konnte rasch begonnen werden.

\section{Zusammenfassung der Ergebnisse}

Buprenorphin stellt nach unserer Einschätzung eine viel versprechende Alternative zum Methadon dar.

In der Entgiftung von Mono-Heroinkonsumenten scheint Methadon/Polamidon ${ }^{\circledR}$ etwas überlegen zu sein. 
Langjährig mit Methadon/Polamidon substituierte Patienten kommen aber mit Buprenorphin in der Entzugsbehandlung von Methadon/Polamidon ${ }^{\circledR}$ deutlich besser zurecht, insbesondere was die Ausprägung von Entzugssymptomen anbelangt (vgl. Abb. 3). Darüber hinaus scheint die Entzugsbehandlung mit Subutex $^{\circledR}$ forcierter als mit ausschleichender Methadon/Polamidon $^{\circledR}$-Dosierung möglich zu sein.

Insbesondere treten nach unseren Beobachtungen deutlich weniger Entzugssymptome nach der letzten Medikamentengabe von Subutex ${ }^{\circledR}$ auf, als bei Polamidon ${ }^{\circledR}$ bzw. Methadon. Dies entspricht der bei anderen Untersuchungen gemachten Erfahrungen [24]. Auch Patienten, die dem Buprenorphin anfangs skeptisch gegenüberstanden, kamen damit subjektiv überraschend gut zurecht und konnten dann dies auch eingestehen.

Schwierigkeiten können in den ersten Tagen der Entgiftungsbehandlung bei der Umstellung auf Buprenorphin auftreten, vor allem bei den langjährig mit Methadon/Polamidon ${ }^{\circledR}$ substituierten Patienten, so dass zu Beginn der Entzugsbehandlung deutlich höher als auch in der Literatur beschrieben, dosiert werden muss (ggf. bis zu 16 oder $20 \mathrm{mg}$ täglich). Diese Erfahrungen finden sich auch in anderen Drogenentzugseinrichtungen wieder [29].

Unter Buprenorphin treten weniger depressive Verstimmungszustände im Gesamtverlauf der Entgiftung auf, als unter der Gabe von Methadon/L-Polamidon ${ }^{\circledR}$. Dies wird auch in anderen Untersuchungen bestätigt [30]. Auch konnte gezeigt werden, dass eine Behandlung der Depressivität bei Substituierten zu einer Verminderung des Opiatbeikonsums beiträgt [31]. Eine Studie von Kosten gelangt sogar zu der Auffassung, dass es sich bei Buprenorphin um ein schnellwirkendes Antidepressivum handelt [4].

Bezüglich der Haltequote haben sich keine auffälligen Unterschiede im Vergleich zur Studie von Bickel et al. [20], die eine bessere Haltequote bei einer Entzugsbehandlung mit Buprenorphin aufwies, zwischen den vier Patientengruppen ergeben.

\section{Literatur}

${ }^{1}$ Bodkin JA, Zornberg GL, Lukas SE et al. Buprenorphine treatment of refractory Depression. Jornal of Clinical Psychopharmacology 1994; 15 49-57

2 Emrich HM, Vogt P, Herz A. Possible antidepressive effects oh opiods: action of Buprenorphine. Annals New York Academy of Sciences 1992; 398: $108-112$

${ }^{3}$ Rance MJ. Animal and molecular pharmacology of mixed agonist-antagonist analgesic drugs. Br J Clin Pharmac 1979; 7: 281S-286S

${ }^{4}$ Kosten et al. (1990): Depressive Symptoms During Buprenorphine Treatment of Opioid Abusers. Journal of Substance Abuse Treatment 1990; $7: 51-54$

${ }^{5}$ Fischer G, Gombas W, Eder $\mathrm{H}$ et al. Vergleichsuntersuchung von Buprenorphin und Methadon im Rahmen der Erhaltungstherapie Opiatkranker. Nervenarzt 1999; 70: 795 -802

${ }^{6}$ Schmauss C, Yassouridis A, Emrich HM. Antipsychotic effect of buprenorphine in schizophrenia. American Journal of Psychiatry 1987; 144 (10): $1340-1342$

7 Dum JE and Herz A. In vivo receptor binding of the opiate antagonist, buprenorphine, correlated with its agonstic and antagonistic actions. Br J Pharmacol 1981; 74: 627-633

${ }^{8}$ Mc Carthy PS and Howlett GJ. Physical dependence induced by opiate partial agonstis. Neuropeptdes 1984; 5: $11-14$
${ }^{9}$ Walsh SL, Preston KL, Stitzer ML et al. Clinical pharmacology of buprenorphine: ceiling effects at high doses. Clin Pharmacol Ther 1994; 55 : $569-580$

10 Doxey JC et al. A comparison of the effects of Buprenorphine and Morphine on the blood gases of conscious rats. J of Pharmacology 1982; 75: 118

${ }^{11}$ Cowan A, Boardman S, Robinson T. Buprenorphine and intestinal motility: a pharma cological analvsis of the biphasic dose response curve. Fred Proc 1977; 36: 994

${ }^{12}$ Kishioka S, Paronis CAl, Lewis JW et al. Buprenorphine and methocloxinnamox, agonist and antagonist effects on respiratory function in rhesus monkeys. European Journal of Pharmacology 2000; 391 : 289-297

${ }^{13}$ Heel RC, Brodgen RN, Speight TM et al. Buprenorphine: a review of ist pharmacological properties and therapeutic efficacy. Drugs 1979; 17: $81-110$

${ }^{14}$ Bickel WK, Stitzer ML, Bigelow GE et al. Buprenorphine: dose-related blockade of opioid challange effects in opioid dependent humans. J. Pharmacol Exp Ther 1988; 247: 47-53

15 Rosen MI, Wallace EA, Mc Mahon TJ et al. Buprenorphine: duration of blockade of effects of intramuscular hydromorphone. Drug Alcohol Depend 1994; 35: 141 - 149

${ }^{16}$ Johnson RE, Risher-Flowers DL, Alim TN et al. Blockade of intravenous hydromorphone $(\mathrm{H})$ by methadone $(\mathrm{M})$ and buprenorphine (B). Clin Pharmacol Ther 1993; 53: 175

17 Cowan A, Lewis JW and Mac Farlane IR. Agonist and antagonist porperties of buprenorphine, a new antinociceptive agent. Br J Pharmacol 1977; 60: 537-545

${ }^{18}$ Arbter PA. Substitution mit Buprenorphin bei HIV-positiven Patienten: Wechselwirkungen mit Proteaseinhibitoren? Suchtmed 2001; 3 (2): $117-118$

${ }^{19}$ Moatti J, Carrieri M, Spire B et al. Moreau and the Manif 2000 study group (2000). Adherence to HAART in French HIV-infected injecting drug users: the contribution of buprenorphine drug maintenance treatment. AIDS 2000; 14: $151-155$

${ }^{20}$ Bickel W, Stitzer M, Bigelow G et al. A clinical trial of Buprenorphin: Comparison with Methadone in the Detoxifikation of Heroin addicts. Clin Pharmacol Ther 1988; 43: $72-78$

21 Johnson RE, Chutuape MA, Strain EC et al. A comparison of levomethadyl acetate, buprenorphine and methadone for opioid dependence. N Engl J Med 2000; 343: 1290-1297

${ }^{22}$ West SL, O`Neal KK, Graham CW. A meta-analysis comparing the effectiveness of buprenorphine and methadone. Journal of substance abuse 2000; 12 : $405-414$

${ }^{23}$ Farre M, Mas A, Torrens $\mathrm{M}$ et al. Retention rate and illicit opioid use during methadone maintenance interventions: a metaanalysis. Drug and Alcohol Dependence 2002; 65: 283-290

24 Janiri L, Mannelli P, Persico AM et al. Opiate detoxifikation of Metadone maintenance patients using lefetamine, clonidine and Buprenorphine. Drug and Alcohol Dependence 1994; 36

${ }^{25}$ Paetzold W, Schneider U, Eronat V et al. Buprenorphin und Carbamazepin in der Entgiftungsbehandlung von Opiatabhängigen mit Drogenbeigebrauch. 7. Suchtmed. Kongressband. Berlin: DGDS 1998; $297-314$

${ }^{26}$ O'Condor PG, Carroll KM, Shi JM et al. Three Methods of opiod Detoxifikation in a Primary Care Setting. 1997

${ }^{27}$ Cheskin LJ, Fudala PJ, Johnson RE. A controlled comparisation of Buprenorphine and Clonidine for acute detoxifikation form opioids. Drug Alcohol Depend 1994; 36 (2): 115-121

${ }^{28}$ Nigam AK, Ray R, Tripathi BM. Buprenorphine in Opiate Withdrawal: A Comparison with Clonidin. Journal of Substance Abuse Treatment 1993; 10: $391-394$

${ }^{29}$ Türbsch L. Stationärer Entzug von und mit Buprenorphin - Abstractband zum Symposium. Buprenorphin - Die neue Alternative zur Methadonsubstitution. Potsdam 1./2.12.2000

${ }^{30}$ Jasinski RE, Cone EJ, Henningfield JE et al. Use of buprenorphin in the treatment of opiate addiction. I. Physiologic and behavioral effects during a rapid dose induction. Clin Pharmacol Ther 1989; 46: 335-343

${ }^{31}$ Paetzold et al. : Buprenorphin: Aspekte der Therapie von Opiatabhängigkeit, Depression und Schizophrenie. Nervenheilkunde 2000; 19: $143-150$ 